\title{
The Uses of Cyclopentanone for the Synthesis of Biologically Active Pyran, Pyridine and Thiophene Derivatives
}

\author{
Eman M. Samir \\ National Organization for Drug Control \& Research, Cairo, Egypt \\ Email: Emanm.samir@yahoo.com
}

Received 12 March 2016; accepted 28 March 2016; published 31 March 2016

Copyright (C) 2016 by author and OALib.

This work is licensed under the Creative Commons Attribution International License (CC BY). http://creativecommons.org/licenses/by/4.0/

(c) (i) Open Access

\begin{abstract}
In the recent work, a series of novel pyran and pyridine and thiophene derivatives were designed and synthesized starting from 2-benzylidenecyclopentanone. The reactivity of these derivatives towards different chemical reagent was studied. The antitumor evaluations of the newly synthesized products were measured and the results showed that some of the synthesized products showed high cytotoxicity.
\end{abstract}

Keywords

Cyclopentanone, Pyran, Pyridine, Pyrimidine

Subject Areas: Medicinal Chemistry

\section{Introduction}

Multicomponent reactions (MCRs), an important subclass of tandem reactions, are one-pot processes in which three or four easily approachable components react to form a single product. The methodology has emerged as a powerful synthetic tool for the preparation of biologically active compounds and important drugs [1] [2]. The multi-component reactions have been used frequently inorganic synthesis, and significant attempts have been focused on the design and development of environmentally friendly and less expensive methods for the generation of libraries of heterocyclic compounds [3] [4]. Therefore, academic and industrial research groups have increasingly focused on the development of MCRs that can lead to new, efficient synthetic methodologies to afford several biologically-active compounds. There has been considerable attention in syntheses reactions and biological activities of $4 \mathrm{H}$-pyran-containing molecules. Furthermore, 4H-pyran derivatives also constitute a structural unit of some pharmaceutical agents, and natural products [5] [6]. The 2-amino-3-cyano-4H-pyran derivatives represent a significant class of compounds, viz. used in cosmetics and pigments and utilized as potentially 
biodegradable agrochemicals [7]. Additionally, several poly functionalized 4H-pyran derivatives have been reported to show a variety of biological activities such as antitumor [8] antibacterial [9] and antimicrobial activities [10]. These compounds are structurally similar to the anticancer agent MX58151 and inhibitors of insulinregulated amino peptidase (IRAP) related to enhancement of memory and learning functions [11] (Figure 1). The 4H-pyran derivatives are also used as photoactive materials [12] and as synthetic intermediates for dihydrofurans [13]. In the present work, we are starting with cyclopentanone as the key starting material for the synthesis of pyran, pyridine, thiophene derivatives together with studying their cytotoxicity against six cancer and one human normal cell lines.

Here in, in order to extend our research on anticancer heterocyclic derivatives with high inhibitory effects toward some cancer cell lines, we report the synthesis of new fused pyran, pyridine, Thiophene derivatives derived from cycloprntanone $\mathbf{1}$. Moreover, some newly synthesized products were good candidates as anticancer drugs through their screening towards cancer and normal cell lines.

\section{Results and Discussion}

The reaction of cyclopentanone with benzaldehyde, 4-nitrobenzaldehyde or 4-methylbenzaldehyde in the presence of piperidine in an oil bath at $120^{\circ} \mathrm{C}$ gave the 2-arylidenecyclopentanone derivatives 3a-c, respectively. The structures of the latter products were based on their respective analytical and spectral data. Thus, the ${ }^{1} \mathrm{H} \mathrm{NMR}$ spectrum of 3c showed $\delta 1.58-2.78\left(\mathrm{~m}, 6 \mathrm{H}, 3 \mathrm{CH}_{2}\right), 3.130\left(\mathrm{~s}, 3 \mathrm{H}, \mathrm{CH}_{3}\right), 7.28-7.39\left(\mathrm{~m}, 4 \mathrm{H}, \mathrm{C}_{6} \mathrm{H}_{4}\right), 7.61(\mathrm{~s}, 1 \mathrm{H}$, $\mathrm{CH}=\mathrm{C}$ ). Compounds 3a-c reacted with malononitrile $\mathbf{4}$ in absolute ethanol containing a catalytic amount of triethylamine gave the pyran derivatives 5a-c, respectively. The analytical and spectral data of the latter products were the basis of their structural elucidation. On the other hand, carrying the same reaction but using ammonium acetate instead of triethylamine gave the pyridine derivatives 6a-c, respectively (Scheme 1).

Next, we studied the reactivity of compounds 3a-c towards thiophene synthesis using the well-known Gewald's thiophene synthesis [14] [15]. Thus, the reaction of either of compounds $\mathbf{3 a}$, $\mathbf{3} \mathbf{b}$ or $\mathbf{3 c}$ with elemental sulfur and malononitrile4 gave the thiophene derivatives $\mathbf{7 a - c}$, respectively. The analytical and spectral data of the latter product are consistent with their respective structures. Thus, the ${ }^{1} \mathrm{HNMR}$ spectrum of compound $7 \mathbf{a}$ showed $\delta 1.52$ - $2.83\left(\mathrm{~m}, 4 \mathrm{H}, 2 \mathrm{CH}_{2}\right), 4.76\left(\mathrm{~s}, 2 \mathrm{H}, \mathrm{D}_{2} \mathrm{O}\right.$ exchangeable, $\left.\mathrm{NH}_{2}\right), 7.21(\mathrm{~s}, 1 \mathrm{H}, \mathrm{CH}=\mathrm{C}), 7.24$ - $7.39(\mathrm{~m}$, $5 \mathrm{H}, \mathrm{C}_{6} \mathrm{H}_{5}$ ). On the other hand, the reaction of either of compound $3 \mathbf{a}, \mathbf{3 b}$ or $\mathbf{3 c}$ with thiourea in an oil bath at $120^{\circ} \mathrm{C}$ gave the 2-(arylidenecyclohexylidene)thiourea derivatives 9a-c, respectively. Moreover, the multi-component reaction (MCR) of any of compound $\mathbf{3 a}$, $\mathbf{3 b}$ or $\mathbf{3} \mathbf{c}$ with thiourea and malononitrile in ethanol containing triethylamine gave the pyrimidine derivatives 10a-c, respectively (Scheme 2). The structures of compounds 10a-c were established on the basis of their analytical and spectral data. Thus, the ${ }^{1} \mathrm{H}$ NMR spectrum of compound 10a showed $\delta 1.39-2.84\left(\mathrm{~m}, 6 \mathrm{H}, 3 \mathrm{CH}_{2}\right), 3.84\left(\mathrm{~s}, 2 \mathrm{H}, \mathrm{CH}_{2}\right), 5.62(\mathrm{~s}, 1 \mathrm{H}, \mathrm{SH}), 6.70(\mathrm{~s}, 1 \mathrm{H}$, pyrimidine $\mathrm{H}-2), 7.28$ - 7.40 (m, 5H, $\left.\mathrm{C}_{6} \mathrm{H}_{5}\right)$.

Next, we studied the reactivity of compounds $\mathbf{3 b}$ and $\mathbf{3 c}$ using ethyl cyanoacetate. Thus, the reaction of either of compound $\mathbf{3 b}$ and $\mathbf{3 c}$ reacted with ethyl cyanoacetate $\mathbf{1 1}$ in ethanol containing a catalytic amount of triethylamine gave the pyran derivatives 12a and 12b, respectively. On the other hand, the reaction of either $\mathbf{3 b}$ or $\mathbf{3 c}$ with ethyl cyanoacetate using ammonium acetate instead of triethylamine gave the pyridine derivatives 13a and 13b, respectively.

The reaction of compound 3c with elemental sulfur and ethyl cyanoacetate $\mathbf{1 1}$ in ethanol containing a catalytic amount of triethylamine gave ethyl4-(methoxybenzylidene)-2-aminoctahydrobenzo[b]thiophene-3-carbo-xylate 14 (Scheme 3).

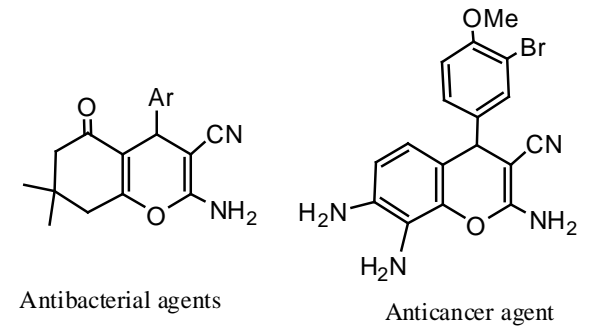<smiles>COc1cc(Br)cc(C2C(C#N)=C(N)Oc3ccccc32)c1</smiles><smiles>N#CC1=C(N)Oc2cc(O)ccc2C1c1cccnc1</smiles>

Figure 1. 2-Amino-3-cyano-4H-pyrans containing heterocycles demonstrating pharmacological and biological activity. 


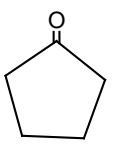

1

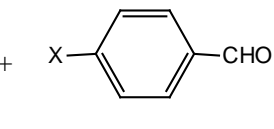

2a, $\mathrm{X}=\mathrm{H}$

b, $\mathrm{X}=4-\mathrm{NO}_{2}$

c, $\mathrm{X}=4-\mathrm{CH}_{3}$

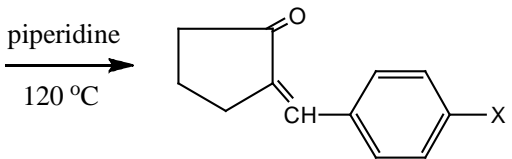

3a, $\mathrm{X}=\mathrm{H}$

b, $\mathrm{X}=4-\mathrm{NO}_{2}$

c, $\mathrm{X}=4-\mathrm{CH}_{3}$<smiles>[X]c1ccc(C2C(C#N)=C(N)OC3=C2CCC3)cc1</smiles>

5a, $\mathrm{X}=\mathrm{H}$

b, $\mathrm{X}=4-\mathrm{NO}_{2}$

c, $\mathrm{X}=4-\mathrm{CH}_{3}$<smiles>[X]c1ccc(C2C(C#N)=C(N)NC3=C2CCC3)cc1</smiles>

6a, $\mathrm{X}=\mathrm{H}$

b, $\mathrm{X}=4-\mathrm{NO}_{2}$

c, $\mathrm{X}=4-\mathrm{CH}_{3}$

\section{Scheme 1. Synthesis of compounds 3a-c; 5a-c and 6a-c.}

\section{Biological Activities}

\subsection{Chemicals}

Fetal bovine serum (FBS) and L-glutamine, were purchased from Gibco Invitrogen Co. (Scotland, UK). RPMI1640 medium was purchased from Cambrex (New Jersey, USA). Dimethyl sulfoxide (DMSO), doxorubicin, penicillin, streptomycin and sulforhodamine B (SRB) were purchased from Sigma Chemical Co. (Saint Louis, USA).

\subsection{Cell Cultures}

The Cell cultures was obtained from the European Collection of cell Cultures (ECACC, Salisbury, UK) and human gastric cancer (NUGC), human colon cancer (DLD1), human liver cancer (HA22T and HEPG2), human breast cancer (MCF), nasopharyngeal carcinoma (HONE1) and normal fibroblast cells (WI38)were kindly provided by the National Cancer Institute (NCI, Cairo, Egypt). They grow as monolayer and routinely maintained in RPMI-1640 medium supplemented with 5\% heat inactivated FBS, $2 \mathrm{mM}$ glutamine and antibiotics (penicillin $100 \mathrm{U} / \mathrm{mL}$, streptomycin $100 \mathrm{lg} / \mathrm{mL}$ ), at $37^{\circ} \mathrm{C}$ in a humidified atmosphere containing $5 \% \mathrm{CO}_{2}$. Exponentially growing cells were obtained by plating $1.5 \times 10^{5}$ cells $/ \mathrm{mL}$ for the six human cancer cell lines including cells derived from $0.75 \times 10^{4}$ cells/mL followed by $24 \mathrm{~h}$ of incubation. The effect of the vehicle solvent (DMSO) on the growth of these cell lines was evaluated in all the experiments by exposing untreated control cells to the maxi- 


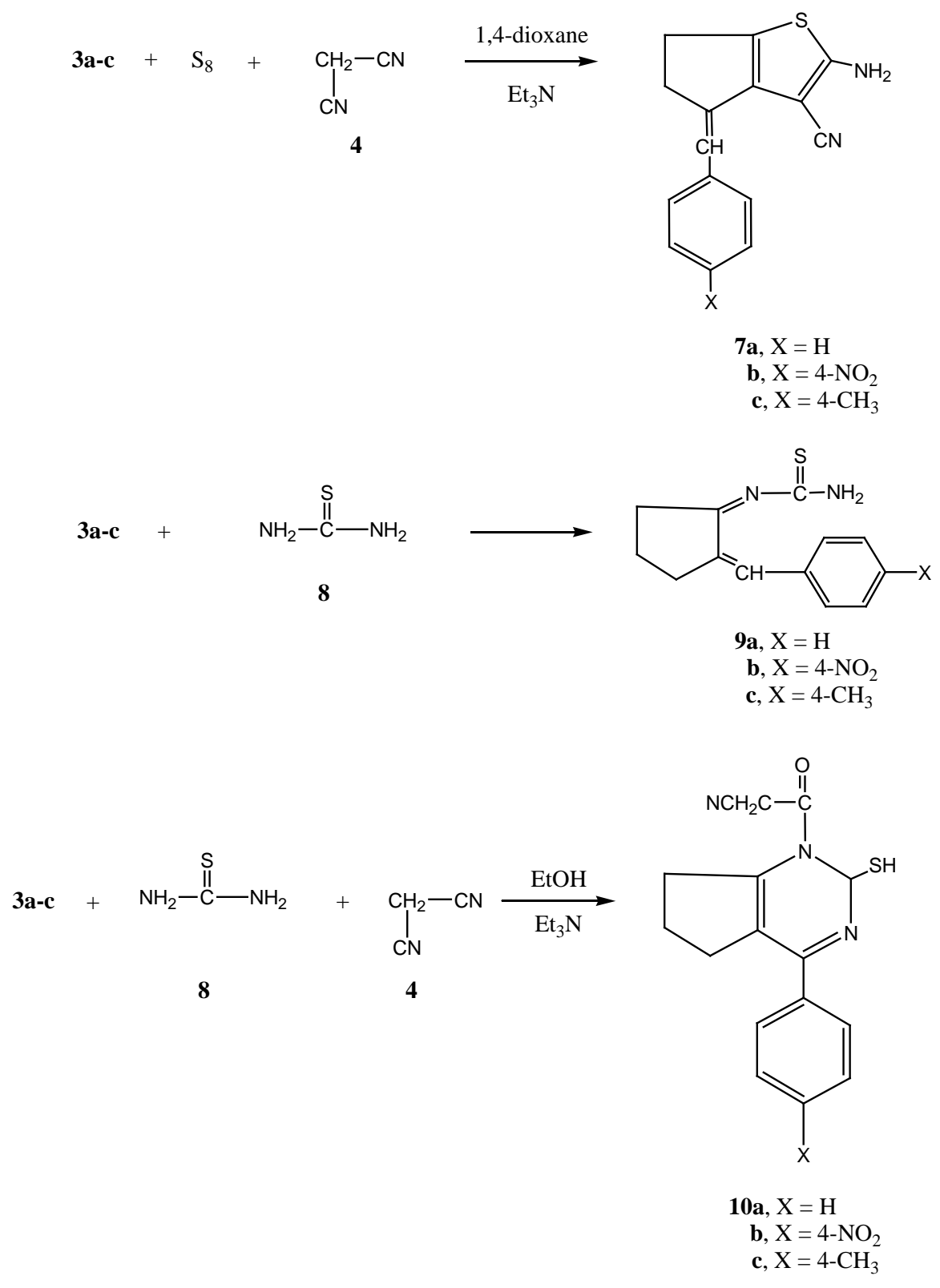

Scheme 2. Synthesis of compounds 7a-c; 9a-c and 10a-c.

mum concentration ( $0.5 \%)$ of DMSO used in each assay.

The heterocyclic compounds, prepared in this study, were evaluated according to standard protocols for their in-vitro cytotoxicity against six human cancer cell lines including cells derived from human gastric cancer (NUGC), human gastric cancer (DLD1), human liver cancer (HA22T and HEPG2), human breast cancer (MCF), nasopharyngeal carcinoma (HONE1) and the normal fibroblast cells (WI38). All of IC $_{50}$ values were listed in Table 1. Some heterocyclic compounds was observed with significant cytotoxicity against most of the cancer cell lines tested $\left(\mathrm{IC}_{50}=10-1000 \mathrm{nM}\right.$ ). Normal fibroblasts cells (WI38) were affected to a much lesser extent (IC50 > 10,000 nM). The cytotoxicity against the tumor cell lines were evaluated through the National cancer Institute in Egypt obeying all ethical rules.

\subsection{Structure Activity Relationship}

From Table 1, it is clear that compounds 5a, 5b, 6c, 7b, 7c, 9c, 10c and 14 are the most potent compounds 

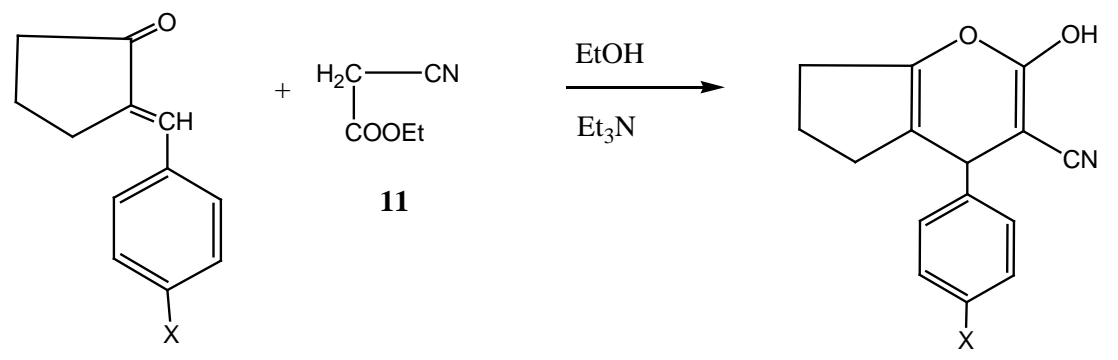

3b, $\mathrm{X}=\mathrm{NO}_{2}$

12a, $\mathrm{X}=\mathrm{NO}_{2}$

c, $\mathrm{X}=\mathrm{CH}_{3}$

b $\mathrm{X}=\mathrm{CH}_{3}$<smiles>[X]c1ccc(C=C2CCCC2=O)cc1</smiles><smiles>CCOC(=O)CC#N</smiles><smiles>CCOC(C)C(N)=O</smiles><smiles>[X]c1ccc(C2C(C#N)=C(O)NC3=C2CCC3)cc1</smiles>

$3 \mathbf{b}, \mathrm{X}=\mathrm{NO}_{2}$

c, $\mathrm{X}=\mathrm{CH}_{3}$

13a, $X=\mathrm{NO}_{2}$

b, $\mathrm{X}=\mathrm{CH}_{3}$

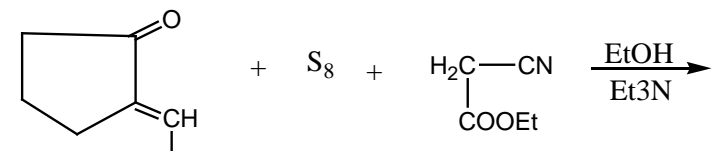<smiles>CCOC(=O)c1c(N)sc2c1C(=Cc1ccc(C)cc1)CC2</smiles>

14

Scheme 3. Synthesis of compounds 12a, 12b, 13a, 13b and 14.

among the tested compounds. It is clear that compounds 3a-c showed low potency. Considering the pyran derivatives 5a-c, it is clear that 5a with the un-substituted phenyl group and 5b with the 4-nitrosubstitutent are more potency than compound 5c with the 4-methyl substituent. On the other hand, for the pyridine derivatives 6a-c the 4-methyl substituent $\mathbf{6 c}$ showed the higher potency than $\mathbf{6 a}$ and $\mathbf{6} \mathbf{b}$. For the thiophene derivatives $\mathbf{7 a - c}$, it is clear that compounds $\mathbf{7 b}$ and $\mathbf{7 c}$ are more potent than 7a. In addition, for compounds $\mathbf{9 a - c}$, it is obvious that the 4-methyl substituted compound $\mathbf{9 c}$ is more potent than $\mathbf{9 b}$ and $\mathbf{9 c}$. The pyrimidine derivatives 10a-c, compound 10c with the 4-nitro substituent showed the highest potency among the three compounds. The pyran 12a, b and pyridines 13a, b derivatives showed low potency toward the six cancer cell lines. The thiophene derivative $\mathbf{1 4}$ showed the maximum potency towards the six cancer cell lines among the tested compounds.

\section{Experimental}

All melting points determined on an Electrothermal digital meltig point apparatus and are uncorrected. IR spec- 
Table 1. Cytotoxicity of novel pregnenlone derivatives against a variety of six human cancer cell lines $\left[\mathrm{IC}_{50}{ }^{\mathrm{b}}\left(\mathrm{nM}^{\mathrm{n}}\right) \mathrm{and}^{\mathrm{a}}\right.$ normal human cell line.

\begin{tabular}{|c|c|c|c|c|c|c|c|}
\hline \multirow{2}{*}{ Compound } & \multicolumn{7}{|c|}{ Cytotoxocity ( IC $_{50}$ in nM) } \\
\hline & NUGC & DLDI & HA22T & HEPG2 & HONE1 & MCF & WI38 ${ }^{\mathrm{c}}$ \\
\hline $3 a$ & 1378 & 2393 & 2768 & 3298 & 2292 & 2472 & $\mathrm{Na}$ \\
\hline $3 \mathbf{b}$ & 3278 & 2283 & 2080 & 2772 & 2630 & 2049 & $\mathrm{Na}$ \\
\hline $3 c$ & 1122 & 1274 & 2366 & 1096 & 1239 & 2145 & $\mathrm{Na}$ \\
\hline $5 a$ & 190 & 105 & 99 & 2389 & 1153 & 2059 & $\mathrm{Na}$ \\
\hline $5 b$ & 44 & 122 & 1764 & 1077 & 1184 & 1662 & $\mathrm{Na}$ \\
\hline $5 c$ & 28 & 1349 & 1884 & 1870 & 1089 & 887 & $\mathrm{Na}$ \\
\hline $6 a$ & 2376 & 2370 & 1259 & 1163 & 983 & 550 & $\mathrm{Na}$ \\
\hline $6 b$ & 2548 & 2210 & 2672 & 1877 & 1603 & 1438 & $\mathrm{Na}$ \\
\hline 6c & 77 & 49 & 42 & 59 & 39 & 1106 & $\mathrm{Na}$ \\
\hline $7 a$ & 3082 & 2180 & 2361 & 2360 & 1672 & 2036 & $\mathrm{Na}$ \\
\hline $7 \mathbf{b}$ & 149 & 113 & 1277 & 1398 & 108 & 92 & $\mathrm{Na}$ \\
\hline 7c & 48 & 1274 & 1449 & 329 & 323 & 120 & $\mathrm{Na}$ \\
\hline $9 a$ & 1321 & 2318 & 1163 & 2318 & 218 & 241 & $\mathrm{Na}$ \\
\hline $9 b$ & 2235 & 2662 & 2187 & 2962 & 1029 & 1392 & $\mathrm{Na}$ \\
\hline 9c & 893 & 1280 & 152 & 627 & 831 & 240 & $\mathrm{Na}$ \\
\hline $10 a$ & 1641 & 2163 & 2117 & 3277 & 3219 & 1986 & $\mathrm{Na}$ \\
\hline $10 \mathrm{~b}$ & 1264 & 1387 & 2218 & 2130 & 2058 & 2342 & $\mathrm{Na}$ \\
\hline $10 \mathrm{c}$ & 34 & 120 & 1276 & 329 & 1432 & 2893 & $\mathrm{Na}$ \\
\hline $12 a$ & 1054 & 1083 & 2383 & 2196 & 1286 & 1142 & $\mathrm{Na}$ \\
\hline $12 b$ & 1039 & 2024 & 1305 & 1440 & 1873 & 1873 & $\mathrm{Na}$ \\
\hline $13 a$ & 1183 & 1082 & 1247 & 1408 & 1662 & 1482 & $\mathrm{Na}$ \\
\hline $13 b$ & 1236 & 1290 & 1157 & 1195 & 1279 & 1243 & $\mathrm{Na}$ \\
\hline 14 & 29 & 41 & 90 & 44 & 32 & 636 & $\mathrm{Na}$ \\
\hline CHS 828 & 25 & 2315 & 2067 & 1245 & 15 & 18 & 378 \\
\hline
\end{tabular}

${ }^{a}$ NUGC, human gastric cancer, DLDI, colon cancer, HA22T, liver cancer, HEPG2, liver cancer; HONEI, nasopharyngeal carcinoma; MCF, breast cancer; WI38, normal fibroblast cells; ${ }^{\mathrm{b}}$ The sample concentration produces a $50 \%$ reduction in cell growth; ${ }^{\mathrm{c}} \mathrm{Na}$ indicating no activity towards the normal cell line.

tra (KBr discs) were recorded on a FTIR plus 460 or Pyeunicam SP-1000 spectrophotometer. ${ }^{1} \mathrm{H}$ NMR spectra were recorded with Mercury-300BB (300 MHz) (Cairo university) instrument in DMSO- $d_{6}$ as solvent using TMS as internal standard and chemical shifts are expressed as $\delta$ ppm.

General procedure for synthesis of 2-benzylidenecyclohexanone derivatives $3 a-c$

Equimolar amounts of 1 (0.84 mL, $0.01 \mathrm{~mol})$ and either benzaldhyde (1.06 g, $0.01 \mathrm{~mol})$, p-nitrobenzaldhyde ( $1.52 \mathrm{~g}, 0.01 \mathrm{~mol})$ or p-methylbenzaldhyde $(1.2 \mathrm{~mL}, 0.01 \mathrm{~mol})$ containing a catalytic amount of piperidine $(0.5$ $\mathrm{mL}$ ) was heated under reflux at $120^{\circ} \mathrm{C}$ for 2 hours. The reaction mixture allowed to cool at room temperature and then poured onto ice/water. The mixture was neutralized by adding few drops of concentrated $\mathrm{HCl}$. The solid productformed was collected by filtration and crystallized from ethanol.

2-(benzylidene)cyclopentanone (3a) 
Yellow crystals, m.p. $76^{\circ} \mathrm{C}$, yield 64\% (1.10 g) IR (KBr) $\left(v\right.$-cm $\left.{ }^{-1}\right): 3056\left(\mathrm{CH}\right.$ aromatic), $2876\left(\mathrm{CH}_{2}\right), 1688$ $(\mathrm{C}=\mathrm{O}), 1536(\mathrm{C}=\mathrm{C}) .{ }^{1} \mathrm{HNMR}\left(\mathrm{DMSO}_{6}, 400 \mathrm{MHZ}\right)(\delta-\mathrm{ppm}): 1.49-2.46\left(\mathrm{~m}, 6 \mathrm{H}, 3 \mathrm{CH}_{2}\right), 7.00-7.67(\mathrm{~m}, 5 \mathrm{H}$, $\mathrm{C}_{6} \mathrm{H}_{5}$ ), 7.56 (s, $1 \mathrm{H}, \mathrm{CH}=\mathrm{C}$ ). Analysis Calcd for $\mathrm{C}_{12} \mathrm{H}_{12} \mathrm{O}$ (172.22): C, 83.69; H, 7.02. Found: C, 83.88; H, 7.29. 2-(4-Nitrobenzylidene) cyclopentanone (3b)

Yellow crystals, m.p. $70^{\circ} \mathrm{C}$, yield 62\% (1.35 g) $\mathrm{IR}(\mathrm{KBr})\left(v-\mathrm{cm}^{-1}\right): 3104,3062\left(\mathrm{CH}\right.$ aromatic), $2988\left(\mathrm{CH}_{2}\right)$, $1669(\mathrm{C}=\mathrm{O}), 1620(\mathrm{C}=\mathrm{C}) .{ }^{1} \mathrm{HNMR}\left(\mathrm{DMSO}_{6}, 400 \mathrm{MHZ}\right)\left(\delta\right.$-ppm): 1.52 - $2.24\left(\mathrm{~m}, 6 \mathrm{H}, 3 \mathrm{CH}_{2}\right), 7.33$ - $7.46(\mathrm{~m}$, $\left.4 \mathrm{H}, \mathrm{C}_{6} \mathrm{H}_{4}\right), 7.50$ (s, $1 \mathrm{H}, \mathrm{CH}=\mathrm{C}$ ). Analysis Calcd for $\mathrm{C}_{12} \mathrm{H}_{11} \mathrm{NO}_{3}$ (217.22): C, 66.35; H, 5.10; N, 6.45. Found: C, 66.82; H, 5.29; N, 6.27.

2-(4-Methylbenzylidene)cyclopentanone (3c)

Yellow crystals, m.p. 58 ${ }^{\circ} \mathrm{C}$, yield 77\% (1.43 g) IR (KBr) $\left(v-\mathrm{cm}^{-1}\right)$ : $3058\left(\mathrm{CH}\right.$ aromatic), $2878\left(\mathrm{CH}_{2}\right), 1690$ $(\mathrm{C}=\mathrm{O}), 1629(\mathrm{C}=\mathrm{C}) .{ }^{1} \mathrm{HNMR}\left(\mathrm{DMSO}_{6}, 400 \mathrm{MHZ}\right)\left(\delta\right.$-ppm): $1.58-2.78\left(\mathrm{~m}, 6 \mathrm{H}, 3 \mathrm{CH}_{2}\right), 3.130\left(\mathrm{~s}, 3 \mathrm{H}, \mathrm{CH}_{3}\right)$, 7.28 - 7.39 (m, $\left.4 \mathrm{H}, \mathrm{C}_{6} \mathrm{H}_{4}\right)$, $7.61(\mathrm{~s}, 1 \mathrm{H}, \mathrm{CH}=\mathrm{C})$. Analysis Calcd for $\mathrm{C}_{13} \mathrm{H}_{14} \mathrm{O}$ (186.25): C, 83.83; H, 7.58. Found: C, 83.62; H, 7.80.

General procedure for synthesis of 2-amino-5,6,7,8-tetrahydro-4-phenyl chromene-3-carbonitrile derivatives $5 a-c$

Equimolar amounts of malononitrile ( $0.66 \mathrm{~g}, 0.01 \mathrm{~mol})$ and $\mathbf{3 a}(1.72 \mathrm{~g}, 0.01 \mathrm{~mol}), 3 \mathbf{b}(2.17 \mathrm{~g}, 0.01 \mathrm{~mol})$ or 3c $(1.86 \mathrm{~g}, 0.01 \mathrm{~mol})$ were dissolved in ethanol $(28 \mathrm{~mL})$ containing a catalytic amount of triethylamine and heated under reflux at $120^{\circ} \mathrm{C}$ for 4 hours. The reaction mixture allowed to cool to room temperature and then poured onto ice/water mixture. The mixture was neutralized by adding a few drops of concentrated HCl. The solid product formed in each case was collected by filtration and crystallized from ethanol.

2-Amino-4-phenyl-4,5,6,7-tetrahydrocyclopenta[b]pyran-3-carbonitrile (5a)

Yellow crystals, m.p. $133^{\circ} \mathrm{C}-136^{\circ} \mathrm{C}$, yield $80 \%(1.90 \mathrm{~g})$. IR $(\mathrm{KBr})\left(v-\mathrm{cm}^{-1}\right): 3459-3323\left(\mathrm{NH}_{2}\right), 3055(\mathrm{CH}$ aromatic), $2978\left(\mathrm{CH}_{2}\right), 2220(\mathrm{CN}) .{ }^{1} \mathrm{H}$ NMR (DMSO-d 6 , $400 \mathrm{MHZ}$ ( $\delta$-ppm): 1.29 - 2.55 (m, 6H, 3CH $), 4.48$ (s, $2 \mathrm{H}, \mathrm{NH}_{2}, \mathrm{D}_{2} \mathrm{O}$ exchangeable), $6.28(\mathrm{~s}, 1 \mathrm{H}$, pyran $\mathrm{H}-4), 7.26-7.39\left(\mathrm{~m}, 5 \mathrm{H}, \mathrm{C}_{6} \mathrm{H}_{5}\right)$. Analysis Calcd for $\mathrm{C}_{15} \mathrm{H}_{14} \mathrm{~N}_{2} \mathrm{O}$ (238.28): C, 75.61; H, 5.92; N, 11.76. Found: C, 75.83; H, 6.29; N, 11.84.

2-Amino-4-(4-nitrophenyl)-4,5,6,7-tetrahydrocyclopenta[b]pyran-3-carbonitrile (5b)

Brown crystals, m.p. $135^{\circ} \mathrm{C}-137^{\circ} \mathrm{C}$, yield $79 \%(2.24 \mathrm{~g}) \mathrm{IR}(\mathrm{KBr})\left(v-\mathrm{cm}^{-1}\right): 3373-3329\left(\mathrm{NH}_{2}\right), 3060(\mathrm{CH}$ aromatic), $2921\left(\mathrm{CH}_{2}\right), 2222(\mathrm{CN}), 1638(\mathrm{C}=\mathrm{C}) .{ }^{1} \mathrm{H}$ NMR (DMSO-d 6 , $\left.400 \mathrm{MHZ}\right)(\delta$-ppm): $1.78-2.29(\mathrm{~m}, 6 \mathrm{H}$, $3 \mathrm{CH}_{2}$ ), $4.68\left(\mathrm{~s}, 2 \mathrm{H}, \mathrm{NH}_{2}\right), 6.78$ (s, $1 \mathrm{H}$, pyran $\left.\mathrm{H}-4\right), 7.25-7.42\left(\mathrm{~m}, 4 \mathrm{H}, \mathrm{C}_{6} \mathrm{H}_{4}\right)$. Analysis Calcd for: $\mathrm{C}_{15} \mathrm{H}_{13} \mathrm{~N}_{3} \mathrm{O}_{3}$ (283.28) Calcd: C, 63.60; H, 4.63; N, 14.83. Found: C. 63.88; H, 4.92; N, 14.68.

2-Amino-4-(p-tolyl)-4,5,6,7-tetrahydrocyclopenta[b]pyran-3-carbonitrile (5c)

Orange crystals, m.p. $168^{\circ} \mathrm{C}-170^{\circ} \mathrm{C}$, yield $88 \%\left(2.22\right.$ g). IR $(\mathrm{KBr})\left(v-\mathrm{cm}^{-1}\right)$ : $3449-3432\left(\mathrm{NH}_{2}\right), 3054$ (CHaromatic), $2210(\mathrm{CN}), 1630(\mathrm{C}=\mathrm{C}) .{ }^{1} \mathrm{H}$ NMR (DMSO-d $\left.{ }_{6}, 400 \mathrm{MHZ}\right)\left(\delta\right.$-ppm): 1.18 - $2.39\left(\mathrm{~m}, 6 \mathrm{H}, 3 \mathrm{CH}_{2}\right), 4.79(\mathrm{~s}$, $2 \mathrm{H}, \mathrm{NH}_{2}, \mathrm{D}_{2} \mathrm{O}$ exchangeable), $3.16\left(\mathrm{~s}, 3 \mathrm{H}, \mathrm{CH}_{3}\right), 6.27$ (s, $1 \mathrm{H}$, pyran $\left.\mathrm{H}-4\right), 7.26$ - $7.43\left(\mathrm{~m}, 4 \mathrm{H}, \mathrm{C}_{6} \mathrm{H}_{4}\right)$. Analysis Calcdfor $\mathrm{C}_{16} \mathrm{H}_{16} \mathrm{~N}_{2} \mathrm{O}$ (252.31): C, 76.16; H, 6.39; N, 11.10. Found: C, 76.29; H, 6.42; N, 10.98.

General procedure for synthesis of cyclopenta[b]pyridinederivatives $(\mathbf{6 a - c})$

Equimolar amount of malononitrile $(0.66 \mathrm{~g}, 0.01 \mathrm{~mol})$ and ammonium acetate $(0.77 \mathrm{~g}, 0.01 \mathrm{~mol})$ in ethanol $(20 \mathrm{~mL})$ was added to either $\mathbf{3 a}(1.72 \mathrm{~g}, 0.01 \mathrm{~mol}), 3 \mathbf{b}(2.17 \mathrm{~g}, 0.01 \mathrm{~mol})$ or $\mathbf{3 c}(1.86 \mathrm{~g}, 0.01 \mathrm{~mol})$. The reaction mixture was heated under reflux at $120^{\circ} \mathrm{C}$ for 4 hours, then allowed to cool to room temperature and poured onto ice/water mixture. The mixture was neutralized by adding few drops of concentrated $\mathrm{HCl}$. The solid products formed was collected by filtration and crystallized from ethanol.

2-Amino-4-phenyl-4,5,6,7-tetrahydro-1H-cyclopenta[b]pyridine-3-carbonitrile (6a)

Reddish brown crystals, m.p. $186^{\circ} \mathrm{C}-189^{\circ} \mathrm{C}$, yield $72 \%(1.71 \mathrm{~g}) . \mathrm{IR}(\mathrm{KBr})\left(v-\mathrm{cm}^{-1}\right)$ : 3468, $3328\left(\mathrm{NH}_{2}, \mathrm{NH}\right)$, 3055 (CHaromatic), $2986\left(\mathrm{CH}_{2}\right), 2220(\mathrm{CN}), 1633$ (C=C). ${ }^{1} \mathrm{H}$ NMR (DMSO-d 6 , $400 \mathrm{MHZ}$ ) ( $\delta$-ppm): 1.38 - 2.59 (m, 6H, 3CH $\mathrm{CH}_{2}$ ), 4.28 (s, $2 \mathrm{H}, \mathrm{D}_{2} \mathrm{O}$ exchangeable, $\left.\mathrm{NH}_{2}\right), 7.19$ (s, $1 \mathrm{H}$, pyridine $\left.\mathrm{H}-4\right), 7.23-7.48\left(\mathrm{~m}, 5 \mathrm{H}, \mathrm{C}_{6} \mathrm{H}_{5}\right)$, 8.32 (s, $1 \mathrm{H}, \mathrm{D}_{2} \mathrm{O}$ exchangeable, NH). ${ }^{13} \mathrm{C}$ NMR (DMSO-d 6 , $75 \mathrm{MHZ}$ ) ( $\delta$-ppm): 28.2, 38.9, $44.05\left(4 \mathrm{CH}_{2}\right), 116.4$ (CN), 119.5, 120.6, 123.6, 124.4, 124.6, 129.2, 133.1, 134.4, $142.6\left(\mathrm{C}_{6} \mathrm{H}_{5}\right.$, pyridine C). Analysis Calcd for $\mathrm{C}_{15} \mathrm{H}_{15} \mathrm{~N}_{3}$ (237.30): C, 75.92; H, 6.37; N, 17.71. Found: C, 76.22; H, 6.28; N, 17.72.

2-Amino-4-(4-nitrophenyl)-4,5,6,7-tetrahydro-1H-cyclopenta[b]pyridine-3-carbonitrile $\mathbf{( 6 b )}$

Yellow crystals, m.p. $166^{\circ} \mathrm{C}-168^{\circ} \mathrm{C}$, yield $79 \%(2.23 \mathrm{~g})$. IR $(\mathrm{KBr})\left(v-\mathrm{cm}^{-1}\right): 3429-3329\left(\mathrm{NH}_{2}, \mathrm{NH}\right), 3060$ (CH aromatic), $2979\left(\mathrm{CH}_{2}\right), 2220(\mathrm{CN}), 1634(\mathrm{C}=\mathrm{C}) .{ }^{1} \mathrm{H}$ NMR (DMSO-d 6 , $\left.400 \mathrm{MHZ}\right)(\delta$-ppm): $1.39-2.70(\mathrm{~m}$, $\left.6 \mathrm{H}, 3 \mathrm{CH}_{2}\right), 4.70\left(\mathrm{~s}, 2 \mathrm{H}, \mathrm{D}_{2} \mathrm{O}\right.$ exchangeable, $\left.\mathrm{NH}_{2}\right), 7.19(\mathrm{~s}, 1 \mathrm{H}$, pyridine $\mathrm{H}-4), 7.26$ - $7.45\left(\mathrm{~m}, 4 \mathrm{H}, \mathrm{C}_{6} \mathrm{H}_{4}\right), 8.42(\mathrm{~s}$, $1 \mathrm{H}, \mathrm{D}_{2} \mathrm{O}$ exchangeable, NH). ${ }^{13} \mathrm{C}$ NMR (DMSO-d $\mathrm{d}_{6}, 75 \mathrm{MHZ}\left(\delta\right.$-ppm): 28.4, 43.6, $45.8\left(4 \mathrm{CH}_{2}\right), 116.3(\mathrm{CN})$, 
120.8, 122.3, 122.9, 123.8, 125.9, 126.7, 129.2, 130.6, $131.6\left(\mathrm{C}_{6} \mathrm{H}_{5}\right.$, pyridine).Analysis Calcd for $\mathrm{C}_{15} \mathrm{H}_{14} \mathrm{~N}_{4} \mathrm{O}_{2}$ (282.30): C, 63.82; H, 5.00; N, 19.85. Found: C, 64.29; H, 5.26; N, 20.16.

2-Amino-4-(p-tolyl)-4,5,6,7-tetrahydro-1H-cyclopenta[b]pyridine-3-carbonitrile (6c)

Yellow crystals, m.p. $155^{\circ} \mathrm{C}-158^{\circ} \mathrm{C}$, yield $80 \%(2.01 \mathrm{~g})$. IR $(\mathrm{KBr})\left(v-\mathrm{cm}^{-1}\right): 3488-3329\left(\mathrm{NH}_{2}, \mathrm{NH}\right), 3055$ (CH aromatic), $2978\left(\mathrm{CH}_{2}\right), 2220(\mathrm{CN}), 1629(\mathrm{C}=\mathrm{C}) .{ }^{1} \mathrm{H}$ NMR (DMSO-d 6 , $\left.400 \mathrm{MHZ}\right)(\delta$-ppm): 1.49 - $2.80(\mathrm{~m}$, $6 \mathrm{H}, 3 \mathrm{CH}_{2}$ ), 3.11 (s, $3 \mathrm{H}, \mathrm{CH}_{3}$ ), 4.72 (s, $2 \mathrm{H}, \mathrm{D}_{2} \mathrm{O}$ exchangeable, $\mathrm{NH}_{2}$ ), 7.11 (s, $1 \mathrm{H}$, pyridine $\left.\mathrm{H}-4\right), 7.24$ - 7.43 (m, $\left.4 \mathrm{H}, \mathrm{C}_{6} \mathrm{H}_{4}\right), 9.4$ (s, $1 \mathrm{H}, \mathrm{D}_{2} \mathrm{O}$ exchangeable, $\mathrm{NH}$ ). Analysis Calcd for $\mathrm{C}_{16} \mathrm{H}_{17} \mathrm{~N}_{3}$ (251.33): C, 76.46; H, 6.82; N, 16.72. Found: C, 76.59; H, 7.04; N, 16.93.

General procedure for synthesis of cyclopenta[b]thiophene-3-carbonitrile derivative (7a-c)

Equimolar amount of malononitrile $(0.66 \mathrm{~g}, 0.01 \mathrm{~mol})$ and elemental sulfur $(0.3 \mathrm{~g}, 0.01 \mathrm{~mol})$ and either $3 \mathbf{a}$ (1.72 g, $0.01 \mathrm{~mol}), 3 \mathbf{b}$ (2.17 g, $0.01 \mathrm{~mol})$ or $3 \mathbf{c}(1.86 \mathrm{~g}, 0.01 \mathrm{~mol})$ were dissolved in 1.4 dioxane $(40 \mathrm{~mL})$ containing a catalytic amount of triethylamine $(0.50 \mathrm{~mL})$. The whole reaction mixture, in each case was heated under reflux for $2 \mathrm{~h}$. The reaction mixture allowed to cool to room temperature and then poured onto ice/water. The mixture was neutralized by adding a few drops of concentrated HCl. Solid products formed was collected by filtration and crystallized from 1,4dioxane.

2-Amino-4-benzylidene-5,6-dihydro-4H-cyclopenta[b]thiophene-3-carbonitrile (7a)

Pale yellow crystals, m.p. $120^{\circ} \mathrm{C}-122^{\circ} \mathrm{C}$, yield $80 \%(2.02 \mathrm{~g})$. IR $(\mathrm{KBr})\left(v-\mathrm{cm}^{-1}\right): 3465-3312\left(\mathrm{NH}_{2}\right), 3058$ (CH aromatic), $2974\left(\mathrm{CH}_{2}\right), 2220(\mathrm{CN}), 1633(\mathrm{C}=\mathrm{C}) .{ }^{1} \mathrm{H}$ NMR (DMSO-d 6 , $\left.400 \mathrm{MHZ}\right)(\delta$-ppm): 1.52 - $2.83(\mathrm{~m}$, $\left.4 \mathrm{H}, 2 \mathrm{CH}_{2}\right), 4.76$ (s, $2 \mathrm{H}, \mathrm{D}_{2} \mathrm{O}$ exchangeable, $\left.\mathrm{NH}_{2}\right), 7.21(\mathrm{~s}, 1 \mathrm{H}, \mathrm{CH}=\mathrm{C}), 7.24-7.39\left(\mathrm{~m}, 5 \mathrm{H}, \mathrm{C}_{6} \mathrm{H}_{5}\right)$. Analysis Calcd for $\mathrm{C}_{15} \mathrm{H}_{12} \mathrm{~N}_{2} \mathrm{~S}$ (252.33): C, 71.40; H, 4.79; N, 11.10; S, 12.71. Found: C, 71.53; H, 4.93; N, 11.52; S, 12.89 .

2-Amino-4-(4-nitrobenzylidene)-5,6-dihydro-4H-cyclopenta[b]thiophene-3-carbonitrile (7b)

Pale yellow crystals, m.p. $177^{\circ} \mathrm{C}-179^{\circ} \mathrm{C}$, yield: $80 \%$ (2.38 g). IR (KBr) $\left(v-\mathrm{cm}^{-1}\right): 3482-3320\left(\mathrm{NH}_{2}\right), 3054$ (CH aromatic), $2991\left(\mathrm{CH}_{2}\right), 2220(\mathrm{CN}), 1632(\mathrm{C}=\mathrm{C}) .{ }^{1} \mathrm{H}$ NMR (DMSO-d $\left.\mathrm{d}_{6}, 400 \mathrm{MHZ}\right)(\delta$-ppm): $1.43-2.87(\mathrm{~m}$, $\left.4 \mathrm{H}, 2 \mathrm{CH}_{2}\right), 4.53\left(\mathrm{~s}, 2 \mathrm{H}, \mathrm{D}_{2} \mathrm{O}\right.$ exchangeable, $\left.\mathrm{NH}_{2}\right), 7.18(\mathrm{~s}, 1 \mathrm{H}, \mathrm{C}=\mathrm{CH}), 7.26-7.39\left(\mathrm{~m}, 4 \mathrm{H}, \mathrm{C}_{6} \mathrm{H}_{4}\right) .{ }^{13} \mathrm{C} \mathrm{NMR}$ (DMSO-d 6 , 75 MHZ ( $\delta$-ppm): 26.2, $35.8\left(2 \mathrm{CH}_{2}\right), 116.3(\mathrm{CN}), 91.3,92.6(\mathrm{CH}=\mathrm{C}), 120.8,122.4,123.1,123.9$, 125.3, 128.4, 129.4, $130.8\left(\mathrm{C}_{6} \mathrm{H}_{5}\right.$, thiophene). Analysis Calcd for $\mathrm{C}_{15} \mathrm{H}_{11} \mathrm{~N}_{3} \mathrm{O}_{2} \mathrm{~S}$ (297.33): C, 60.59; H, 3.73; N, 14.13; S, 10.78. Found: C, 60.72; H, 3.94; N, 14.06; S, 10.88.

2-Amino-4-(4-methylbenzylidene)-5,6-dihydro-4H-cyclopenta[b]thiophene-3-carbonitrile (7c)

Orange crystals, m.p. $188^{\circ} \mathrm{C}-191^{\circ} \mathrm{C}$, yield: $77 \%\left(2.05\right.$ g). IR (KBr) $\left(v-\mathrm{cm}^{-1}\right)$ : $3429-3313\left(\mathrm{NH}_{2}\right), 3054(\mathrm{CH}$ aromatic), $2979\left(\mathrm{CH}_{2}\right), 2220(\mathrm{CN}), 1629(\mathrm{C}=\mathrm{C}) .{ }^{1} \mathrm{H}$ NMR (DMSO-d $\left.{ }_{6}, 400 \mathrm{MHZ}\right)(\delta$-ppm): $1.39-2.87(\mathrm{~m}, 4 \mathrm{H}$, $2 \mathrm{CH}_{2}$ ), 3.13 (s, $3 \mathrm{H}, \mathrm{CH}_{3}$ ), 4.84 (s, $2 \mathrm{H}, \mathrm{D}_{2} \mathrm{O}$ exchangeable, $\mathrm{NH}_{2}$ ), $7.21(\mathrm{~s}, 1 \mathrm{H}, \mathrm{C}=\mathrm{CH}), 7.27-7.39\left(\mathrm{~m}, 4 \mathrm{H}, \mathrm{C}_{6} \mathrm{H}_{4}\right)$. Analysis Calcd for $\mathrm{C}_{16} \mathrm{H}_{14} \mathrm{~N}_{2} \mathrm{~S}$ (266.36) C, 72.15; H, 5.30; N, 10.52; S, 12.04. Found: C, 71.88; H, 5.42; N, 10.31; S, 11.93.

General procedure for synthesis of 2-cyclopentylidene) thiourea derivatives (9a-c)

Equimolar amount of thiourea ( $0.76 \mathrm{~g}, 0.01 \mathrm{~mol})$, either of $3 \mathbf{a}(1.72 \mathrm{~g}, 0.01 \mathrm{~mol}), 3 \mathbf{b}(2.17 \mathrm{~g}, 0.01 \mathrm{~mol})$ or $3 \mathbf{c}$ $(1.86 \mathrm{~g}, 0.01 \mathrm{~mol})$ were dissolved in ethanol $(25 \mathrm{~mL})$ containing a catalytic amount of triethylamine and heated under reflux for 2 hours. The reaction mixture allowed to cool to room temperature and then poured onto ice/ water mixture. The mixture was neutralized by adding few drops of concentrated HCl. The solid product formed was collected by filtration, crystallized from ethanol.

2-Benzylidene cyclopentylidenethiourea $(\mathbf{9 a})$

Yellow crystals, m.p. $137^{\circ} \mathrm{C}-139^{\circ} \mathrm{C}$, yield $73 \%(1.60 \mathrm{~g})$. IR $(\mathrm{KBr})\left(v-\mathrm{cm}^{-1}\right)$ : $3467-3324\left(\mathrm{NH}_{2}\right), 3055(\mathrm{CH}$ aromatic), $2983\left(\mathrm{CH}_{2}\right), 1630(\mathrm{C}=\mathrm{C}) .{ }^{1} \mathrm{H}$ NMR $\left(\delta\right.$-ppm): $1.44-2.73\left(\mathrm{~m}, 6 \mathrm{H}, 3 \mathrm{CH}_{2}\right), 4.49\left(\mathrm{~s}, 2 \mathrm{H}, \mathrm{D}_{2} \mathrm{O}\right.$ exchangeable, $\mathrm{NH}_{2}$ ), 7.05 (s, $\left.1 \mathrm{H}, \mathrm{C}=\mathrm{CH}\right), 7.25-7.41\left(\mathrm{~m}, 5 \mathrm{H}, \mathrm{C}_{6} \mathrm{H}_{5}\right) .{ }^{13} \mathrm{C}$ NMR (DMSO-d 6 , $75 \mathrm{MHZ}(\delta$-ppm): 28.2, 45.8, 46.2 $\left(4 \mathrm{CH}_{2}\right)$, 89.3, 90.6 $(\mathrm{CH}=\mathrm{C}), 120.8,121.3,125.2,127.8\left(\mathrm{C}_{6} \mathrm{H}_{5}\right), 167.2(\mathrm{C}=\mathrm{S}), 173.1(\mathrm{C}=\mathrm{N})$. Analysis Calcd for $\mathrm{C}_{13} \mathrm{H}_{14} \mathrm{~N}_{2} \mathrm{~S}$ (230.33): C, 67.79; H, 6.13; N, 12.16; S, 13.92. Found: C, 67.84; H, 5.83; N, 11.92; S, 14.11 .

4-Nitrobenzylidene)cyclopentylidenethiourea $(\mathbf{9 b})$

Orange crystals, m.p. $244^{\circ} \mathrm{C}-248^{\circ} \mathrm{C}$, yield: $66 \%(1.82 \mathrm{~g})$. IR $(\mathrm{KBr})\left(v-\mathrm{cm}^{-1}\right): 3480-3322\left(\mathrm{NH}_{2}\right), 3060(\mathrm{CH}$ aromatic), $2979\left(\mathrm{CH}_{2}\right), 1620(\mathrm{C}=\mathrm{C}) .{ }^{1} \mathrm{H}$ NMR (DMSO-d $\left.\mathrm{d}_{6}, 400 \mathrm{MHZ}\right)\left(\delta\right.$-ppm): $1.39-2.88\left(\mathrm{~m}, 6 \mathrm{H}, 3 \mathrm{CH}_{2}\right), 4.72$ (s, $\left.2 \mathrm{H}, \mathrm{NH}_{2}\right), 7.16(\mathrm{~s}, 1 \mathrm{H}, \mathrm{CH}=\mathrm{C}), 7.22-7.67\left(\mathrm{~m}, 4 \mathrm{H}, \mathrm{C}_{6} \mathrm{H}_{4}\right)$. Analysis Calcd for $\mathrm{C}_{13} \mathrm{H}_{13} \mathrm{~N}_{3} \mathrm{O}_{2} \mathrm{~S}(275.33): \mathrm{C}, 56.71$; H, 4.76; N, 15.26; S, 11.65. Found: C, 56.53; H, 4.93; N, 15.42; S, 11.82.

2-(4-Methylbenzylidene)cyclohexylidenethiourea $(9 \mathrm{c})$

Brown crystals, m.p. $220^{\circ} \mathrm{C}-223^{\circ} \mathrm{C}$, yield: 67\% (1.63 g). IR $(\mathrm{KBr})\left(v-\mathrm{cm}^{-1}\right): 3480-3329\left(\mathrm{NH}_{2}\right), 3045(\mathrm{CH}$ 
Aromatic), $2975\left(\mathrm{CH}_{2}\right), 1629(\mathrm{C}=\mathrm{C}) .{ }^{1} \mathrm{H}$ NMR (DMSO-d 6 , $\left.400 \mathrm{MHZ}\right)\left(\delta\right.$-ppm): $1.40-2.69$ (m, 6H, 3CH $\mathrm{CH}_{2}$ ), 3.10 (s, $\left.3 \mathrm{H}, \mathrm{CH}_{3}\right), 4.60$ (s, $2 \mathrm{H}, \mathrm{D}_{2} \mathrm{O}$ exchangeable, $\left.\mathrm{NH}_{2}\right), 7.18(\mathrm{~s}, 1 \mathrm{H}, \mathrm{CH}=\mathrm{C}), 7.23-7.41\left(\mathrm{~m}, 4 \mathrm{H}, \mathrm{C}_{6} \mathrm{H}_{4}\right.$ ), Analysis Calcd for $\mathrm{C}_{14} \mathrm{H}_{16} \mathrm{~N}_{2} \mathrm{~S}$ (244.36): C, 68.81; H, 6.60; N, 11.46; S, 13.12. Found: C, 68.93; H, 6.73; N, 11.58; S, 13.49.

General procedure for synthesis of 3-(2-mercapto-2,5,6,7-tetrahydro-1H-cyclopenta[d]pyrimidin-1-yl)-3-oxopropane nitrile derivatives (10a-c)

Equimolar amount of thiourea $(0.76 \mathrm{~g}, 0.01 \mathrm{~mol})$, malononitrile $(0.66 \mathrm{~g}, 0.01 \mathrm{~mol})$ and any of $3 \mathrm{a}(1.72 \mathrm{~g}, 0.01$ $\mathrm{mol})$, $3 \mathbf{b}(2.17 \mathrm{~g}, 0.01 \mathrm{~mol})$, or $3 \mathbf{c}(1.86 \mathrm{~g}, 0.01 \mathrm{~mol})$ were dissolved in ethanol $(25 \mathrm{~mL})$ containing a catalytic amount of triethylamine and heated under reflux for $5 \mathrm{~h}$. The reaction mixture allowed to cool to room temperature and then poured onto ice/water mixture. The mixture was neutralized by adding few drops of concentrated $\mathrm{HCl}$. The solid product formed upon cooling was collected by filtration and crystallized from ethanol.

3-(2-Mercapto-4-phenyl-2,5,6,7-tetrahydro-1H-cyclopenta[d]pyrimidin-1-yl)-3-oxopropanenitrile (10a)

Yellow crystals, m.p. $180^{\circ} \mathrm{C}-183^{\circ} \mathrm{C}$, yield: $69 \%(2.05 \mathrm{~g})$. IR (KBr) $\left(v-\mathrm{cm}^{-1}\right): 3058(\mathrm{CH}$ aromatic), 2984 $\left(\mathrm{CH}_{2}\right), 2222(\mathrm{CN}), 1697(\mathrm{C}=\mathrm{O}), 1645(\mathrm{C}=\mathrm{N}), 1630(\mathrm{C}=\mathrm{C}) .{ }^{1} \mathrm{H}$ NMR (DMSO-d $\left.\mathrm{d}_{6}, 400 \mathrm{MHZ}\right)(\delta$-ppm): $1.39-2.84$ (m, $\left.6 \mathrm{H}, 3 \mathrm{CH}_{2}\right), 3.84\left(\mathrm{~s}, 2 \mathrm{H}, \mathrm{CH}_{2}\right), 5.62(\mathrm{~s}, 1 \mathrm{H}, \mathrm{SH}), 6.70(\mathrm{~s}, 1 \mathrm{H}$, pyrimidine $\mathrm{H}-2), 7.28-7.40\left(\mathrm{~m}, 5 \mathrm{H}, \mathrm{C}_{6} \mathrm{H}_{5}\right)$. Analysis Calcd for $\mathrm{C}_{16} \mathrm{H}_{15} \mathrm{~N}_{3} \mathrm{OS}$ (297.37): C, 64.62; H, 5.08; N, 14.13; S, 10.78. Found: C, 64.91; H, 5.26; N, 14.37; S, 10.94.

3-(2-Mercapto-4-(4-nitrophenyl)-2,5,6,7-tetrahydro-1H-cyclopenta[d]-pyrimidin-1-yl)-3-oxopropanenitrile $(\mathbf{1 0 b})$

Yellow crystals, m.p. $194^{\circ} \mathrm{C}-196^{\circ} \mathrm{C}$, yield: $93 \%(3.18 \mathrm{~g})$. IR $(\mathrm{KBr})\left(v-\mathrm{cm}^{-1}\right)$ : $3056(\mathrm{CH}$ aromatic), 2893 $\left(\mathrm{CH}_{2}\right), 2220(\mathrm{CN}), 1690(\mathrm{C}=\mathrm{O}), 1644(\mathrm{C}=\mathrm{N}), 1631(\mathrm{C}=\mathrm{C}) .{ }^{1} \mathrm{H}$ NMR (DMSO-d $\left.\mathrm{d}_{6}, 400 \mathrm{MHZ}\right)(\delta$-ppm): $1.49-2.83$ (m, $\left.6 \mathrm{H}, 3 \mathrm{CH}_{2}\right), 3.85\left(\mathrm{~s}, 2 \mathrm{H}, \mathrm{CH}_{2}\right), 4.62(\mathrm{~s}, 1 \mathrm{H}, \mathrm{SH}), 6.03(\mathrm{~s}, 1 \mathrm{H}$, pyrimidine $\mathrm{H}-2), 7.21-7.44\left(\mathrm{~m}, 4 \mathrm{H}, \mathrm{C}_{6} \mathrm{H}_{4}\right)$. Analysis Calcd for $\mathrm{C}_{16} \mathrm{H}_{14} \mathrm{~N}_{4} \mathrm{O}_{3} \mathrm{~S}$ (342.37): C, 56.13; H, 4.12; N, 16.36; S, 9.37. Found: C, 56.22; H, 4.32; N, 16.08; S, 9.28.

3-(2-Mercapto-4-(p-tolyl)-2,5,6,7-tetrahydro-1H-cyclopenta[d]pyrimidin-1-yl)-3-oxopropanenitrile (10c)

Orange brown crystals, m.p. $177^{\circ} \mathrm{C}-179^{\circ} \mathrm{C}$, yield: $85 \%(2.64 \mathrm{~g})$. IR $(\mathrm{KBr})\left(v-\mathrm{cm}^{-1}\right)$ : $3060(\mathrm{CH}$ aromatic), $2987\left(\mathrm{CH}_{2}\right), 2221(\mathrm{CN}), 1669(\mathrm{C}=\mathrm{O}), 1645(\mathrm{C}=\mathrm{N}), 1630(\mathrm{C}=\mathrm{C}) .{ }^{1} \mathrm{H}$ NMR $\left(\delta\right.$-ppm): $1.39-2.82\left(\mathrm{~m}, 6 \mathrm{H}, 3 \mathrm{CH}_{2}\right)$, 3.14 (s, 3H, $\mathrm{CH}_{3}$ ), 3.48 (s, $\left.2 \mathrm{H}, \mathrm{CH}_{2}\right), 4.62$ (s, $\left.1 \mathrm{H}, \mathrm{SH}\right), 6.30$ (s, $1 \mathrm{H}$, pyrimidine $\left.\mathrm{H}-2\right), 7.24-7.49\left(\mathrm{~m}, 4 \mathrm{H}, \mathrm{C}_{6} \mathrm{H}_{4}\right)$. Analysis Calcd for: $\mathrm{C}_{17} \mathrm{H}_{17} \mathrm{~N}_{3} \mathrm{OS}$ (311.40): C, 65.57; H, 5.50; N, 13.49; S, 10.30. Found: C, 65.77; H, 5.39; N, 13.72; S, 10.26 .

General procedure for synthesis of 2-hydroxy-4,5,6,7-tetrahydro-1H-cyclopenta[b]pyran-3-carbonitrile derivatives $(\mathbf{1 2 a}, \boldsymbol{b})$

Equimolar amount of ethyl 2-cyanoacetate $(1.13 \mathrm{~mL}, 0.01 \mathrm{~mol}) \mathbf{3 b}(2.17 \mathrm{~g}, 0.01 \mathrm{~mol})$, or $3 \mathbf{c}(1.86 \mathrm{~g}, 0.01 \mathrm{~mol})$ were dissolved in ethanol $(25 \mathrm{~mL})$ containing a catalytic amount of triethylamine and heated under reflux for 45 $\min$ in first case and for 3 hours in second case. The reaction mixture allowed to cool to room temperature and then poured onto ice/water mixture. The mixture was neutralized by adding concentrated $\mathrm{HCl}$. The solid product formed was collected by filtration, crystallized from ethanol.

2-Hydroxy-4-(4-nitrophenyl)-4,5,6,7-tetrahydrocyclopenta[b]pyran-3-carbonitrile (12a)

Yellow crystal, m.p. $180^{\circ} \mathrm{C}-183^{\circ} \mathrm{C} .69 \%(1.96 \mathrm{~g})$. IR (KBr) $\left(v-\mathrm{cm}^{-1}\right)$ : $3328(\mathrm{OH}), 3055$ (CH aromatic), 2977 $\left(\mathrm{CH}_{2}\right), 2220(\mathrm{CN}), 1632(\mathrm{C}=\mathrm{C}) .{ }^{1} \mathrm{H}$ NMR (DMSO-d 6 , $\left.400 \mathrm{MHZ}\right)\left(\delta\right.$-ppm): $1.51-2.83\left(\mathrm{~m}, 6 \mathrm{H}, 3 \mathrm{CH}_{2}\right), 5.80(\mathrm{~s}$, $1 \mathrm{H}$, pyran $\mathrm{H}-4), 7.25-7.39\left(\mathrm{~m}, 4 \mathrm{H}, \mathrm{C}_{6} \mathrm{H}_{4}\right), 10.29$ (s, $1 \mathrm{H}, \mathrm{D}_{2} \mathrm{O}$ exchangeable, $\left.\mathrm{OH}\right)$. Analysis Calcd for $\mathrm{C}_{15} \mathrm{H}_{12} \mathrm{~N}_{2} \mathrm{O}_{4}$ (284.27): C, 63.38; H; 4.25; N, 9.85. Found: C, 63.49; H, 4.33; N, 9.59.

2-Hydroxy-4-(p-tolyl)-4,5,6,7-tetrahydro-1H-cyclopenta[b]pyran-3-carbonitrile (12b)

Yellow crystals, m.p. $111^{\circ} \mathrm{C}-113^{\circ} \mathrm{C}$. yield $80 \%(2.02 \mathrm{~g})$. IR (KBr) $\left(v-\mathrm{cm}^{-1}\right): 3544-3329(\mathrm{OH}), 3055(\mathrm{CH}$ aromatic), $2980\left(\mathrm{CH}_{2}\right), 2210(\mathrm{CN}) .{ }^{1} \mathrm{H}$ NMR (DMSO-d 6 , $\left.400 \mathrm{MHZ}\right)\left(\delta\right.$-ppm): 1.41 - 2.65 (m, 6H, 3CH $\left.\mathrm{CH}_{2}\right), 3.11$ (s, $\left.3 \mathrm{H}, \mathrm{CH}_{3}\right), 7.03(\mathrm{~s}, 1 \mathrm{H}$, pyran $\mathrm{H}-4), 7.26-7.58\left(\mathrm{~m}, 4 \mathrm{H}, \mathrm{C}_{6} \mathrm{H}_{4}\right), 10.22(\mathrm{~s}, 1 \mathrm{H}, \mathrm{OH})$. Analysis Calcd for $\mathrm{C}_{16} \mathrm{H}_{15} \mathrm{NO}_{2}$ (253.30): C, 75.87; H, 5.97; N, 5.53. Found: C, 75.58; H, 6.21; N, 5.80.

General procedure for synthesis of 4-1,4,5,6,7,8-hexahydroquinoline-3-carbonitrile (13a,b)

Equimolar amounts of ethyl 2-cyanoacetate $(1.13 \mathrm{~mL}, 0.01 \mathrm{~mol})$ and any of 2-(4-nitrobenzylidene) cyclopentanone $(2.17 \mathrm{~g}, 0.01 \mathrm{~mol})$ or 2-(4-Methylbenzylidene) cyclopentanone $(1.86 \mathrm{~g}, 0.01 \mathrm{~mol})$ were dissolved in ethanol $(25 \mathrm{~mL})$ containing catalytic amount of ammonium acetate $(0.77 \mathrm{gm}, 0.01 \mathrm{~mol})$ and heated under reflux at $100^{\circ} \mathrm{C}$ for 2 hours. The reaction mixture allowed to cool to room temperature and then poured onto ice/water mixture. The mixture was neutralized by adding few drops of concentrated $\mathrm{HCl}$. The solid product formed was 
collected by filtration, crystallized from ethanol.

2-Hydroxy-4-(4-nitrophenyl)-4,5,6,7-tetrahydro-1H-cyclopenta[b]pyridine-3-carbonitrile (13a)

Yellow crystals, m.p. $210^{\circ} \mathrm{C}-214^{\circ} \mathrm{C}$. yield: $70 \%(1.98 \mathrm{~g})$. IR(KBr) $\left(v-\mathrm{cm}^{-1}\right): 3522-3312(\mathrm{OH}, \mathrm{NH}), 3051$ (CH aromatic), $2986\left(\mathrm{CH}_{2}\right), 2220(\mathrm{CN}), 1634(\mathrm{C}=\mathrm{C}) .{ }^{1} \mathrm{H}$ NMR (DMSO-d $\left.\mathrm{d}_{6}, 400 \mathrm{MHZ}\right)(\delta$-ppm): $1.49-2.80(\mathrm{~m}$, $6 \mathrm{H}, 3 \mathrm{CH}_{2}$ ), 5.99 (s, $1 \mathrm{H}$, pyridineH-4), 7.30-7.39 (m, $\left.4 \mathrm{H}, \mathrm{C}_{6} \mathrm{H}_{4}\right), 8.29$ (s, $1 \mathrm{H}, \mathrm{D}_{2} \mathrm{O}$ exchangeable, $\left.\mathrm{NH}\right), 10.22(\mathrm{~s}$, $1 \mathrm{H}, \mathrm{D}_{2} \mathrm{O}$ exchangeable, OH). ${ }^{13} \mathrm{C}$ NMR (DMSO-d $\mathrm{d}_{6}, 75 \mathrm{MHZ}\left(\delta\right.$-ppm): 26.9, 41.4, $44.3\left(3 \mathrm{CH}_{2}\right), 116.7(\mathrm{CN})$, 120.2, 121.4, 123.1, 125.3, 125.8, 126.2, 129.4, 155.8, $157.3\left(\mathrm{C}_{6} \mathrm{H}_{4}\right.$, pyran).Analysis Calcdfor $\mathrm{C}_{15} \mathrm{H}_{13} \mathrm{~N}_{3} \mathrm{O}_{3}$ (283.28): C, 63.60; H; 4.63; N, 14.83. Found: C, 63.49; H, 4.74; N, 15.02.

2-Hydroxy-4-(p-tolyl)-4,5,6,7-tetrahydro-1H-cyclopenta[b]pyridine-3-carbonitrile (13b)

Yellow light crystals, m.p. $160^{\circ} \mathrm{C}$. yield: $85 \%(2.14 \mathrm{~g})$. IR $(\mathrm{KBr})\left(v-\mathrm{cm}^{-1}\right): 3442-3315(\mathrm{OH}, \mathrm{NH}), 3003(\mathrm{CH}$ aromatic), $2937-2830\left(\mathrm{CH}_{2}\right), 2210(\mathrm{CN}) .{ }^{1} \mathrm{H}$ NMR (DMSO-d $\left.\mathrm{d}_{6}, 400 \mathrm{MHZ}\right)\left(\delta\right.$-ppm): $1.23-1.57\left(\mathrm{~m}, 6 \mathrm{H}, 3 \mathrm{CH}_{2}\right)$, 3.81 (s, $3 \mathrm{H}, \mathrm{CH}_{3}$ ), 7.00 (s, $1 \mathrm{H}$, pyridine $\left.\mathrm{H}-4\right), 7.00-7.58\left(\mathrm{~m}, 4 \mathrm{H}, \mathrm{C}_{6} \mathrm{H}_{4}\right), 8.29$ (s, $1 \mathrm{H}, \mathrm{D}_{2} \mathrm{O}$ exchangeable, $\mathrm{NH}$ ), 10.22 (s, $1 \mathrm{H}, \mathrm{D}_{2} \mathrm{O}$ exchangeable, OH). ${ }^{13} \mathrm{C}$ NMR (DMSO-d 6 , $\left.75 \mathrm{MHZ}\right)\left(\delta\right.$-ppm): 30.1, 45.6, 49.5, $50.5\left(4 \mathrm{CH}_{2}\right)$, $96.07\left(\mathrm{OCH}_{3}\right), 117.6(\mathrm{CN}), 120.5,120.6,124.6,129.2,129.8,133.1,134.5,140.3,145.2\left(\mathrm{C}_{6} \mathrm{H}_{4}\right.$, pyridine $\left.\mathrm{C}\right)$. Analysis Calcd for $\mathrm{C}_{16} \mathrm{H}_{16} \mathrm{~N}_{2} \mathrm{O}(252.31)$ : C, 76.16; H, 6.39; N, 11.10. Found: C, 76.26; H, 6.42; N, 10.84 .

Synthesis of ethyl4-(methoylbenzylidene)-2-amino-octa-hydrobenzo-[b]thiophene-3-carboxylate (14)

Equimolar amount of 2-(4-methylbenzylidene) cyclopentanone 3c (1.86 g, $0.01 \mathrm{~mol})$, elementals sulfur (0.32 g, $0.01 \mathrm{~mol})$ and ethyl 2-cyanoacetate $(1.16 \mathrm{~mL}, 0.01 \mathrm{~mol})$ were dissolved in ethanol $(20 \mathrm{~mL})$ containing catalytic amount of triethylamine and heated under reflux for $2 \mathrm{~h}$. The reaction mixture allowed to cool to room temperature and then poured onto ice/water mixture. The mixture was neutralized by adding few drops of concentrated $\mathrm{HCl}$. The solid product formed was collected by filtration, crystallized from ethanol.

Yellow crystals, m.p. $222^{\circ} \mathrm{C}-225^{\circ} \mathrm{C}$, yield: $77 \%(2.53 \mathrm{~g})$. IR $(\mathrm{KBr})\left(v-\mathrm{cm}^{-1}\right): 3469-3319\left(\mathrm{NH}_{2}\right), 3059(\mathrm{CH}$ aromatic), $2986\left(\mathrm{CH}_{2}\right), 1703(\mathrm{C}=\mathrm{O}), 1610(\mathrm{C}=\mathrm{C}) .{ }^{1} \mathrm{H}$ NMR (DMSO-d 6 , $\left.400 \mathrm{MHZ}\right)(\delta$-ppm): $1.13(\mathrm{t}, 3 \mathrm{H}, \mathrm{J}=$ $\left.7.22 \mathrm{~Hz}, \mathrm{CH}_{3}\right), 2.49-2.59$ (m, 4H, 2CH $), 3.09$ (s, $\left.3 \mathrm{H}, \mathrm{CH}_{3}\right), 4.21$ (q, $\left.2 \mathrm{H}, \mathrm{J}=7.22 \mathrm{~Hz}, \mathrm{CH}_{2}\right), 4.29\left(\mathrm{~s}, 2 \mathrm{H}, \mathrm{D}_{2} \mathrm{O}\right.$ exchangeable, $\mathrm{NH}_{2}$ ), 7.21 - $7.38\left(\mathrm{~m}, 5 \mathrm{H}, \mathrm{CH}=\mathrm{C}, \mathrm{C}_{6} \mathrm{H}_{4}\right.$ ). Analysis Calcd for $\mathrm{C}_{18} \mathrm{H}_{19} \mathrm{NO}_{2} \mathrm{~S}$ (313.41): C, 68.98; $\mathrm{H}$, 6.11; N, 4.47; S, 10.23. Found: C, 68.57; H, 6.04; N, 4.49; S, 9.89.

\section{Conclusions}

Our results showed that the electronegative $\mathrm{NO}_{2}$ and $\mathrm{CN}$ hydrophobic groups in the Compounds might play a very important role in enhancing the cytotoxic effect.

In summary, we have developed a convenient synthetic approach to 26 samples. The regioselective attack by different reagents on the active center moiety led to the diversity of the produced systems, CHNS Elemental analyses, IR, ${ }^{1} \mathrm{H}$ NMR spectral data. The cytotoxicity of the newly synthesized products showed that the thiophene derivative $\mathbf{1 4}$ showed the maximum cytotoxicity among the tested compounds.

\section{Acknowledgements}

E. M. Samir would like to express her deepest that to Professor Rafat M. Mohareb, Professor of Organic Chemistry at Cairo University for his kind revision of this work.

\section{References}

[1] Bahman, S., Mahmoodi, N.O., Mamaghani, M., Tabatabaeian, K., Chirani, A.S. and Nikokar, I. (2013) Facile Regioselective Synthesis of Novel Bioactive Thiazolyl-Pyrazoline Derivatives via a Three-Component Reaction and Their Antimicrobial Activity. Bioorganic \& Medicinal Chemistry Letters, 23, 548-551. http://dx.doi.org/10.1016/j.bmcl.2012.11.024

[2] Hosseinnia, R., Mamaghani, M., Tabatabaeian, K., Shirini, F. and Rassa, M. (2012) An Expeditious Regioselective Synthesis of Novel Bioactive Indole-Substituted Chromene Derivatives via One-Pot Three-Component Reaction. Bioorganic \& Medicinal Chemistry Letters, 22, 5956-5960. http://dx.doi.org/10.1016/j.bmcl.2012.07.059

[3] ]Foroughifar, N. and Ebrahimi, S. (2013) One-Pot Synthesis of 1,3-Thiazolidin-4-One Using Bi( $\left.\mathrm{SCH}_{2} \mathrm{COOH}\right)_{3}$ as Catalyst. Chinese Chemical Letters, 24, 389-391.

[4] Baharfar, R. and Baghbanian, S.M. (2012) Synthesis of Novel Uracil Based 2, 5-Diaminofuransusing Multi-Component Reactions. Chinese Chemical Letters, 23, 677-680. http://dx.doi.org/10.1016/j.cclet.2012.04.011

[5] Bonsignore, L., Loy, G., Secci, D. and Calignano, A. (1993) Synthesis and Pharmacological Activity of 2-Oxo-(2H) 1- 
Benzopyran-3-Carboxamide Derivatives. European Journal of Medicinal Chemistry, 28, 517-520. http://dx.doi.org/10.1016/0223-5234(93)90020-F

[6] Tietze, L.F. (1983) Secologanin, a Biogenetic Key Compound-Synthesis and Biogenesis of the Iridoid and Secoiridoid Glycosides. Angewandte Chemie International Edition, 22, 828-841.

[7] Hafez, E.A.A., Elnagdi, M.H., Elagamey, A.G.A., et al. (1987) Nitriles in Heterocyclic Synthesis: Novel Synthesis of Benzo[c]coumarin and of benzo[c]pyrano[3, 2-c]quinoline Derivatives. Heterocycles, 26, 903-907. http://dx.doi.org/10.3987/R-1987-04-0903

[8] Hong, H., Huang, L.J. and Teng, D.W. (2011) A Spirocyclic Oxindole Analogue: Synthesis and Antitumor Activities. Chinese Chemical Letters, 22, 1009-1012.

Wang, D.-C., Xie, Y.-M., Fan, C., et al. (2014) Efficient and Mild Cyclization Procedures for the Synthesis of Novel 2Amino-4H-pyran Derivatives with Potential Antitumor Activity. Chinese Chemical Letters, 25, 1011-1013. http://dx.doi.org/10.1016/j.cclet.2014.04.026

[9] Kumar, D., Reddy, V.B., Sharad, S., et al. (2009) A Facile One-Pot Green Synthesis and Antibacterial Activity of 2Amino-4H-pyrans and 2-amino-5-oxo-5, 6, 7, 8-tetrahydro-4H-chromenes. European Journal Medicinal Chemistry, 44, 3805-3809. http://dx.doi.org/10.1016/j.ejmech.2009.04.017

[10] Sangani, C.B., Mungra, D.C., Patel, M.P., et al. (2012) Synthesis and in Vitro Antimicrobial Screening of New Pyrano[4, 3-b]pyran Derivatives of 1H-Pyrazole. Chinese Chemical Letters, 23, 57-60. http://dx.doi.org/10.1016/j.cclet.2011.09.012

[11] Kemnitzer, W., Kasibhatla, S., Jiang, S., et al. (2005) Discovery of 4-Aryl-4H-chromenesas a New Series of Apoptosis Inducers Using a Cell- and Caspase-Based High Throughput Screening Assay. 2. Structure-Activity Relationships of the 7- and 5-, 6-, -8, Positions. Bioorganic \& Medical Chemistry Letters, 15, 4745-4751. http://dx.doi.org/10.1016/j.bmcl.2005.07.066

Kasibhatla, S., Gourdeau, H., Meerovitch, K., et al. (2004) Discovery and Mechanism of Action of a Novel Series of Apoptosis Inducers with Potential Vascular Targeting Activity. Molecular Cancer Therapeutics, 3, 1365-1374.

[12] Armesto, D., Horspool, W.M., Martin, N., et al. (1989) Synthesis of Cyclobutenes by the Novel Photochemical Ring Contraction of 4-Substituted 2-amino-3, 5-dicyano-6-phenyl-4H-pyrans. Journal of Organic Chemistry, 54, 3069-3072. http://dx.doi.org/10.1021/jo00274a021

[13] Chennapuram, M., Emmadi, N.R., Bingi, C., et al. (2014) Group-Assisted Purification (GAP) Chemistry for Dihydrofurans: Water as a Medium for Catalyst Free Synthesis in a One Pot Four Component Reaction. Green Chemistry, 16, 3237-3246.

[14] Puterová, Z., Krutošíková, A. and Véghc, D. (2010) Gewald Reaction Synthesis, Properties and Applications of Substituted 2-Aminothiophenes. ARKIVOC, 2010, 209-246. http://dx.doi.org/10.3998/ark.5550190.0011.105

[15] Buchstaller, H.P., Siebert, C.D., Lyssy, R.H., Frank, I., Duran, A., Gottschlich, R. and Noe, C.R. ( 2001) Synthesis of Novel 2-Aminothiophenes-3-carboxylate by Variations of the Gewald Reaction. Monatshefte für Chemie, 132, 279-293. http://dx.doi.org/10.1007/s007060170137 